\title{
Wanted: guidelines that doctors will follow
}

\author{
Implementation is the problem
}

Imagine a world where every patient received the best known treatment. Now identify the barriers to realising such a Utopian ideal. Many doctors would nominate "resources" and happily leave it at that. But, as a comparison of health care systems across the world shows, spending more money is not the only answer. Ignorance, incompetence, poor management, and a sometimes deliberate disregard of established knowledge all get in the way of best practice. ${ }^{1}$ Strategies other than increasing resources are therefore needed.

Governments are becoming increasingly interested in what these other strategies might be. For example, in Britain medical audit, Health of the Nation, and the NHS's research and development programme are best understood as different answers to the same question: how can we improve the outcome of care, given roughly the same resources?

Currently, it is guidelines' turn under the spotlight. Last month the Scottish Office published Clinical Guidelines, ${ }^{1}$ and last week the Clinical Outcomes Group of the Department of Health sponsored a workshop on the topic. Terminology is proving a sticking point. Here Clinical Guidelines provides the following serviceable definitions: "Clinical guidelines are systematically developed statements which assist in decision making about appropriate health care for specific clinical conditions. It is recommended that the term 'clinical guideline' should apply to the general statement of principle and that the word 'protocol' should cover the more detailed development of these broad principles for local application."

But there is a bigger sticking point than terminology: implementation. Many more guidelines have been drawn up than have been implemented. And so many and so complicated are the barriers to implementation that it is arguable whether any more guidelines should be drawn up until some of these barriers have been surmounted.

Here are a few of them. Guidelines are intellectually suspect: by reflecting expert opinion they may formalise unsound practice. Meant to tackle variations in practice, they risk standardising practice around the average, which is not necessarily the best. They may stifle innovation (and hence progress) and prevent discretion in individual cases. Legal anxieties about negligence abound. As Clinical Guidelines points out, negligence arises when practitioners fall below the standards of care expected of their competent colleagues: if guidelines purport to embody these standards then justifying deviations from them would be difficult. Some fear that "cookbook medicine" will reduce doctors' self respect and could reduce patients' confidence in them. Some critics of guidelines believe that clinical freedom, like other sorts of freedom, cannot be limited without being lost.

These are not trivial problems: they strike at the heart of what it means to be a doctor. If doctors are not required to exercise judgment what are they there for? Sooner or later the great debate between individual judgment and agreed standards will have to be held. It is not all uncharted territory ahead: Clinical Guidelines reminds us that "Clinical freedom implies the obligation to do what is best for the patient at all times, not the right to do whatever one pleases." Guidelines would spell the end of surgery as "playschool for adults," as a surgeon at the workshop described it. Would anyone other than surgeons lose out if they lost the right to make idiosyncratic decisions?

Given all these problems, it is unsurprising that clinical guidelines have the best chance of succeeding if they are developed by those who will be using them. This suggests an obvious solution to who should draw them up, but the experience of the Professional Standard Review Organisation in the United States is instructive on this point. It estimated that some $\$ 100 \mathrm{~m}$ was spent by individual hospitals drawing up practice guidelines for patients with myocardial infarction. Despite this investment it produced "no library from which a set of criteria for treating a patient with a heart attack can be checked out.... Doing it right might have cost $\$ 150000$ to $\$ 500000$ per condition and no single institution had that kind of money available."2 The solution now favoured by Clinical Guidelines and speakers at the workshop is to have scientifically validated guidelines set at a national or regional level, which are modified for local use. Plans for implementing guidelines will usually require more thought and effort than does drawing them up.

Far from saving money in the short term, guidelines will require investment for their development and implementation. To fulfil their aim they will have to be constantly updated. Clinical Guidelines warns that though a reduction in costs may be a byproduct of ensuring the most effective use of resources, optimal practice may, on occasion, be more expensive than current practice. It is a complicated equation: "Any consideration of the cost of developing guidelines should take into account the likely cost of not developing, or not implementing them, in terms of harm to patients, waste of resources, and exposure to medico-legal risks."

At the workshop Dr Graham Winyard, medical director of the NHS Management Executive, made explicit the financial context in which guidelines were being considered. $\mathrm{He}$ reminded participants that the Treasury was interested in whether current medical interventions and the substantial variations in clinical outcomes could be justified. He wondered whether the "incredibly powerful lever of contracting" could have some part to play in getting doctors to follow guidelines (preferably by the next financial year). A trust chairman noted wryly that previously insuperable difficulties with guidelines had disappeared when contracts were at stake. Clinical Guidelines concludes that "incentives and sanctions are a major area of uncertainty ... which will need to be explored if clinical guidelines are to be brought into general use."

While we await the results of explorations into these and other tricky areas two recent developments are worth noting. In response to the widely varying quality of existing guidelines Robert Hayward and colleagues have proposed a structured abstract for descriptions of practice guidelines, designed "to help readers obtain the key information needed to assess the applicability, importance, and validity of any guideline." And the Cochrane Centre, which has found that doctors have as much difficulty using the results of systematic reviews as they do following guidelines, is setting up a group focusing on behavioural change. This seems the logical place to start. As with systematic reviews, resources have been poured into guidelines but almost no attention has been paid to doctors' reluctance to follow them.

TONY DELAMOTHE Deputy editor

\section{$B M \mathcal{Y}$}

1 Clinical Resource and Audit Group. Clinical guidelines. Edinburgh: Scottish Office, 1993.

2 Brook RH. Practice guidelines and practising medicine. $3 A M A$ 1989;262:3027-31.

3 Hayward RSA, Wilson MC, Tunis SR, Bass EB, Rubin HR, Haynes B. More informative abstracts of articles describing clinical practice guidelines. Ann Intern Med 1993;118:731-7. 